\title{
Entropy and Exact Matrix-Product Representation of the Laughlin Wave Function
}

\author{
S. Iblisdir, ${ }^{1}$ J. I. Latorre, ${ }^{1}$ and R. Orús ${ }^{1,2}$ \\ ${ }^{1}$ Departament d'Estructura i Constituents de la Matèria, Universitat de Barcelona, 647 Diagonal, 08028 Barcelona, Spain \\ ${ }^{2}$ School of Physical Sciences, University of Queensland, QLD 4072, Australia
}

(Received 14 September 2006; published 6 February 2007)

\begin{abstract}
An analytical expression for the von Neumann entropy of the Laughlin wave function is obtained for any possible bipartition between the particles described by this wave function, for a filling fraction $\nu=1$. Also, for a filling fraction $\nu=1 / m$, where $m$ is an odd integer, an upper bound on this entropy is exhibited. These results yield a bound on the smallest possible size of the matrices for an exact representation of the Laughlin ansatz in terms of a matrix-product state. An analytical matrix-product state representation of this state is proposed in terms of representations of the Clifford algebra. For $\nu=1$, this representation is shown to be asymptotically optimal in the limit of a large number of particles.
\end{abstract}

DOI: 10.1103/PhysRevLett.98.060402

The puzzling behavior of a cold gas of electrons confined in two spatial dimensions, and subjected to the effect of transverse magnetic fields, is the origin of two of the most studied phenomena in condensed matter physics: the integer and the fractional quantum Hall effects [1,2]. While it is possible to give a satisfactory explanation for the properties of the integer quantum Hall effect with filling fraction $\nu=1$, by means of transport and related models [3], a complete understanding of the fractional case is still missing. It is commonly believed that the interactions between the particles are essentially responsible for this situation. A relevant approach, in this respect, is the Laughlin ansatz for the wave function of the ground state of the system [4]. While so far this state has only been proven to be an exact eigenstate of very specific Hamiltonians [5] and for some specific values of the filling fraction, it contains the relevant properties that the ground state of the real system must have. The Laughlin Wave Function (LWF) has also been turned to be a valuable ansatz in describing the physics of rapidly rotating small atomic Bose-Einstein condensates [6]. However, to check that the LWF is indeed the ground state of the fractional quantum Hall effect seems to be a difficult (computational) problem that has, so far, only been solved for systems made of a small number of particles, typically of the order of 10 [5].

In this Letter, we wish to provide some clues on why this problem is so difficult, in the light of recent developments in the fields of quantum information science and manybody physics. It is now known that a profound relation exists between the difficulty to simulate numerically a quantum system in a given state and the von Neumann entropy between parts of the system this state exhibits, which can be understood in terms of the so-called Matrix-Product States (MPS) [7]. With this relation in mind, we have computed the bipartite von Neumann entropy of a system as described by the Laughlin ansatz $(\nu=$ 1). As we shall discuss, this computation is instructive in understanding the nature of the correlations exhibited by a system in such a state. To our knowledge, the behavior of the von Neumann entropy was known only for the case of
PACS numbers: 05.30.-d, 03.75.Kk, 03.75.Lm, 03.75.Ss

one particle versus the rest and in very specific situations [8]. In addition, we will provide an MPS representation for this state, which is optimal in the thermodynamical limit. This representation will then be extended to some noninteger values of the filling fraction $\nu$.

The LWF for $n$ particles and filling fraction $\nu$ reads [4]

$$
\Psi_{\nu}\left(z_{1}, \ldots, z_{n}\right)=\mathcal{N}_{\nu}(n) \prod_{i<j}\left(z_{i}-z_{j}\right)^{1 / \nu} \prod_{i=1}^{n} e^{-\left|z_{i}\right|^{2} / 2},
$$

where $z_{j}, j=1, \ldots, n$ stands for the position of the particle $j$ in the $x-y$ plane written as a single complex coordinate $z_{j}=x_{j}+i y_{j}$. Note that, but in a few cases, the computation of the normalization constant, $\mathcal{N}_{\nu}(n)$ seems to be a very difficult problem [9].

It is convenient to introduce an orthonormal monoparticle basis defined as

$$
\phi_{a}\left(z_{i}\right)=\frac{1}{\sqrt{\pi a !}} z_{i}^{a} e^{-\left|z_{i}\right|^{2} / 2} \quad a=0, \ldots, n-1 .
$$

Using this basis, the LWF, for $\nu=1$, can be expressed as a Slater determinant:

$$
\Psi_{1}\left(z_{1}, \ldots, z_{n}\right)=\frac{1}{\sqrt{n !}} \sum_{a_{1}, \ldots, a_{n}=0}^{n-1} \epsilon^{a_{1} \ldots a_{n}} \phi_{a_{1}}\left(z_{1}\right) \ldots \phi_{a_{n}}\left(z_{n}\right),
$$

where $\epsilon$ denotes the Levi-Civita completely antisymmetric tensor in $n$ dimensions, with the convention $\epsilon^{0,1, \ldots, n-1}=$ -1 .

The above expressions show that it is possible to correctly describe the quantum state by means of a local Hilbert space of dimension $n$ for each particle. Since we have $n$ particles, the total dimension of the complete Hilbert space scales then as $n^{n}$. This superexponential scaling is at the heart of the difficulty to validate the ansatz (1) by means of an exact diagonalization [5].

Let us now compute the von Neumann entropy for any subset of $k$ particles for a system of $n$ indistinguishable particles in the state $\Psi_{1}\left(z_{1}, \ldots, z_{n}\right)$. Note that this 
von Neumann entropy cannot be interpreted as the number of distillable EPR pairs. Because of the symmetrization, it is impossible to associate a label with the particles and perform the appropriate distillation operations.

The reduced density matrix for a subset of $k$ particles out of $n$ reads

$$
\begin{aligned}
\rho_{k, n} \equiv & \rho_{k, n}\left(w_{1}, \ldots, w_{k} ; z_{1}, \ldots, z_{k}\right) \\
= & \int d z_{k+1} \ldots d z_{n} \Psi_{1}^{*}\left(w_{1}, \ldots, w_{k}, z_{k+1}, \ldots, z_{n}\right) \\
& \times \Psi_{1}\left(z_{1}, \ldots, z_{k}, z_{k+1}, \ldots, z_{n}\right) .
\end{aligned}
$$

From the orthonormality of the monoparticle basis, we get

$$
\begin{aligned}
\rho_{k, n}= & \frac{1}{n !} \sum_{a, b, c} \boldsymbol{\epsilon}^{a_{1} \ldots a_{k} c_{k+1} \ldots c_{n}} \boldsymbol{\epsilon}^{b_{1} \ldots b_{k} c_{k+1} \ldots c_{n}} \\
& \times \phi_{a_{1}}^{*}\left(w_{1}\right) \phi_{b_{1}}\left(z_{1}\right) \ldots \phi_{a_{k}}^{*}\left(w_{k}\right) \phi_{b_{k}}\left(z_{k}\right) .
\end{aligned}
$$

This density matrix can be written in a diagonal form by introducing the following set of orthonormal basis for the subset of $k$ particles:

$$
\Phi_{c}(z) \equiv \frac{1}{\sqrt{k !}} \epsilon^{a_{1} \ldots a_{k} c_{k+1} \ldots c_{n}} \phi_{a_{1}}\left(z_{1}\right) \ldots \phi_{a_{k}}\left(z_{k}\right),
$$

where $(z) \equiv\left(z_{1}, \ldots, z_{k}\right)$ and where the indices are sorted such that $c_{k+1}<\ldots<c_{n}$ and, thus, the combined index $c$ ranges from 1 to $\left(\begin{array}{l}n \\ k\end{array}\right)$. It is then possible to see that all eigenvalues of $\rho_{k, n}$ are identical, that is

$$
\rho_{k, n}=\frac{1}{\left(\begin{array}{l}
n \\
k
\end{array}\right)} \sum_{c=1}^{\left(\begin{array}{c}
n \\
k
\end{array}\right)} \Phi_{c}^{*}(w) \Phi_{c}(z) .
$$

The von Neumann entropy then reads

$$
S_{k, n} \equiv-\operatorname{Tr}\left(\rho_{k, n} \log _{2} \rho_{k, n}\right)=\log _{2}\left(\begin{array}{l}
n \\
k
\end{array}\right),
$$

and gets its maximum value for a bipartition of the system into two pieces of equal number of particles:

$$
S_{k, n} \leq S_{(n / 2), n} \sim n-\frac{1}{2} \log _{2} \frac{n \pi}{2} .
$$

Equation (9) shows that the effective dimension of the Hilbert space for half a Laughlin gas is $O\left(2^{n}\right)$, which is a weaker scaling than the naive growth, $O\left(n^{n / 2}\right)$, of the Hilbert space for $n / 2$ particles.

Let us now perform a similar computation in the case $\nu=1 / m$, when $m=2 s+1$ is an odd positive integer different from 1. As explained in Ref. [9], $\Psi_{\nu}\left(z_{1}, \ldots, z_{n}\right)$ can be expanded in terms of mutually orthogonal Slater determinants:

$$
\Psi_{\nu}\left(z_{1} \ldots z_{n}\right)=\prod_{i=1}^{n} e^{-\left|z_{i}\right|^{2} / 2} \times \sum_{l_{1}, \ldots, l_{n}} g_{l_{1} \ldots l_{n}}^{(s)}\left|\begin{array}{ccc}
z_{1}^{l_{1}} & \ldots & z_{1}^{l_{n}} \\
\vdots & \vdots & \vdots \\
z_{n} & \ldots & z_{n}
\end{array}\right|,
$$

where the indices $l_{1}, \ldots, l_{n}$ are constrained by $0 \leq$ $l_{1}<\ldots<l_{n} \leq(2 s+1)(n-1)$.

Let $A(n, s)$ denote the number of coefficients in this expansion. The rank $\tau_{k}$ of the reduced density matrix of any subset of $k$ particles is bounded as

$$
\tau_{k} \leq A(n, s)\left(\begin{array}{l}
n \\
k
\end{array}\right) .
$$

Indeed, as we have seen in the study of the case $\nu=1,\left(\begin{array}{l}n \\ k\end{array}\right)$ is the Schmidt rank of each of the individual Slater determinants, and the Schmidt rank of a sum of kets is smaller than or equal to the sum of the ranks of individual kets. As abundantly discussed in Ref. [9], to give a closed form for $A(n, s)$ seems to be a very hard problem. Fortunately, an upper bound on $A(n, s)$ can be derived as follows. Because of the constraints between the indices, the expansion (10) does not feature more than $[(2 s+1)(n-1)+1]^{n}$ terms. This trivial bound could be reached, would each index be allowed to range from 0 to $(2 s+1)(n-1)$ independently from the values taken by the other indices. But one can further constraint $A(n, s)$ : since a given permutation of the columns of a determinant produces the same determinant (up to a sign factor), we have

$$
A(n, s) \leq[(2 s+1)(n-1)+1]^{n} / n !
$$

Therefore, for $k=n / 2$ and in the large $n$ limit,

$$
\tau_{n / 2} \leq \frac{(2 s+1)^{n} n^{n}}{n^{n}} 2^{n}=(4 s+2)^{n},
$$

and the von Neumann entropy then obeys

$$
S_{(n / 2), n} \leq n(\log m+\log 2) .
$$

This bound can actually be slightly improved upon using the fact that all indices $l_{0}, \ldots, l_{n-1}$ assume different values.

Let us now derive explicit MPS representations of the LWF, starting with the case $\nu=1$. The dimensions of the matrices in an MPS representation of a given quantum state are related to the von Neumann entropy of bipartitions of the system when the particles it is made of are ordered on a line [7]. According to our previous calculation, an MPS representation of the LWF in terms of matrices of size $O\left(2^{n}\right)$ should be possible:

$$
\begin{aligned}
\Psi_{\nu=1}\left(z_{1} \ldots z_{n}\right)= & \frac{1}{\sqrt{n !}} \sum_{a_{1}, \ldots, a_{n}=0}^{n-1} \operatorname{Tr}\left(A^{[1] a_{1}} \ldots A^{[n] a_{n}}\right) \\
& \times \phi_{a_{1}}\left(z_{1}\right) \ldots \phi_{a_{n}}\left(z_{n}\right),
\end{aligned}
$$

where the matrix $A^{[i] a_{i}}$ is associated with the particle $i$ being in the monoparticle state $\phi_{a_{i}}$. These matrices have a size which is the same for all values of $i, a_{i}: \chi \times \chi$. We will now see that the properties of Clifford algebras are well suited in order to find these matrices. The Clifford algebra $\mathrm{Cl}(0, n)$ is defined by

$$
\left\{\gamma^{a}, \gamma^{b}\right\}=2 \delta^{a b} \quad a, b=0, \ldots, n-1,
$$


where each matrix $\gamma_{\alpha \beta}^{a}$ has indices $\alpha, \beta=1, \ldots, \chi$ (see, for example, [10]).

Let us start with the case where $n$ is even. The representation theory of the Clifford algebra dictates that $\chi=$ $2^{n / 2}$. The matrices $\gamma_{\alpha \beta}^{a}$ provide the following MPS construction:

$$
\epsilon^{a_{1} \ldots a_{n}}=\frac{-1}{(2 i)^{n / 2}} \operatorname{Tr}\left(\gamma^{a_{1}} \ldots \gamma^{a_{n}} \gamma_{5}\right)
$$

where $\gamma_{5} \equiv(-i)^{n / 2} \gamma^{0} \ldots \gamma^{n-1}$. This result emerges from the basic trace properties of the matrices $\gamma_{5}$ and $\gamma^{a}, a=$ $0, \ldots, n-1$, and shows that all the matrices $A^{[i] a_{i}}$ can be taken the same for all particles but one. For example:

$$
A^{[i] a_{i}} \equiv \gamma^{a_{i}} \quad i=1, \ldots, n-1, \quad A^{[n] a_{n}} \equiv \gamma^{a_{n}} \gamma_{5} .
$$

Our construction shows that, in spite of the symmetry of the system, the MPS representation is not made of a set of identical matrices.

The MPS construction (18) is asymptotically optimal in the sense that the matrices have, in the limit of large $n$, the minimal size. To begin with, let us first compute how large should the matrices be in an open boundary MPS representation. Given a bipartition of the system into a "left" and a "right" part, corresponding, respectively, to the $k$ first and $n-k$ last particles of the bipartition, the state (15) can be rewritten as

$$
\sum_{i, j} \sum_{\alpha=1}^{\chi} L_{1, \alpha}^{(i)} R_{\alpha, 1}^{(j)} \varphi_{i}^{L}\left(z_{1}, \ldots, z_{k}\right) \varphi_{j}^{R}\left(z_{k+1}, \ldots, z_{n}\right),
$$

where $\varphi_{i}^{L}$ (resp. $\varphi_{j}^{R}$ ) denotes some pure state for the left (resp. right) part of the system. The latter state (19) can be rewritten as $\sum_{\alpha=1}^{\chi} \tilde{\varphi}_{\alpha}^{L}\left(z_{1}, \ldots, z_{k}\right) \tilde{\varphi}_{\alpha}^{R}\left(z_{k+1}, \ldots, z_{n}\right)$, so the rank of the density matrix of the left (or right) system is bounded by $\chi$. Therefore, the maximal von Neumann entropy for that bipartition is $S_{\max }=\log _{2} \chi$.

In the case of a periodic boundary representation, the state (15) is rewritten as $\sum_{\alpha, \beta=1}^{\chi} \tilde{\varphi}_{\alpha, \beta}^{L}\left(z_{1}, \ldots, z_{k}\right) \times$ $\varphi_{\alpha, \beta}^{R}\left(z_{k+1}, \ldots, z_{n}\right)$, so that the rank of either reduced density matrix is now bounded by $\chi^{2}$. Therefore, if $S$ denotes the actual von Neumann entropy of the bipartition, we have

$$
\chi \geq 2^{S / 2} \text { periodic boundary MPS. }
$$

The effective rank of a bipartition has contributions coming from the two borders. As we have already seen, in our case the maximum possible entropy over all bipartitions corresponds to $S_{(n / 2), n} \sim n$, which in turn implies that the dimension of the matrices of the Clifford algebra precisely matches the lower bound provided by this entropy for large $n$

$$
\chi=2^{(1 / 2) S_{(n / 2), n}} \sim 2^{n / 2} .
$$

Therefore, the representation (18) is optimal in the limit of large $n$ values.
Explicit representation of the MPS construction can be obtained from the chain of isomorphisms

$$
\mathrm{Cl}(0, n+2) \approx \mathrm{Cl}(0,2) \otimes \mathrm{Cl}(0, n) .
$$

For $n=2$, set $\gamma^{0}=\sigma^{x}, \gamma^{1}=\sigma^{y}$ and $\gamma_{5}=\sigma^{z}$. A representation for $n+2$ is constructed from a representation of $n$ as follows:

$$
\begin{aligned}
& \gamma_{(n+2)}^{i}=1 \otimes \gamma_{(n)}^{i}, \quad i=0, \ldots, n-1, \\
& \gamma_{(n+2)}^{i}=\gamma_{(2)}^{i-n} \otimes \gamma_{5,(n)}, \quad i=n, n+1 .
\end{aligned}
$$

A representation of the Clifford algebra for the case $n$ odd can be simply derived from a representation for $n-1$, upon taking

$$
\gamma_{(n)}^{i}=\gamma_{(n-1)}^{i}, \quad i=0, \ldots, n-2, \quad \gamma_{(n)}^{n-1}=\gamma_{5(n-1)} .
$$

Then, it can be shown that $\gamma_{5(n)}=(-)^{n} i^{3 / 2}$ and that the antisymmetric tensor is given by

$$
\epsilon^{a_{1} \ldots a_{n}}=\frac{-i^{1 / 2}}{(2 i)^{(n-1) / 2}} \operatorname{Tr}\left(\gamma^{a_{1}} \ldots \gamma^{a_{n}}\right) .
$$

It is also possible to extend the MPS construction for $\nu=1$ to $\nu=\frac{1}{m}$, where $m$ is an integer. The basic idea is to use the property of the product of traces $\operatorname{Tr}\left(A^{1} \ldots A^{n}\right) \operatorname{Tr}\left(B^{1} \ldots B^{n}\right)=\operatorname{Tr}\left[\left(A^{1} \otimes B^{1}\right) \ldots\left(A^{n} \otimes B^{n}\right)\right]$ in a recursive way. Let us exemplify the construction in the case of $1 / \nu=2$, (bosonic statistics).

Up to a global prefactor and a set of particle-dependent factors, the problem of finding a faithful MPS representation essentially reduces to representing

$$
\begin{aligned}
& \operatorname{Tr}\left(\gamma^{a_{1}^{(1)}} \ldots \gamma^{a_{n}^{(1)}} \gamma_{5}\right) \operatorname{Tr}\left(\gamma^{a_{1}^{(2)}} \ldots \gamma^{a_{n}^{(2)}} \gamma_{5}\right) \\
& \quad \times \phi_{a_{1}^{(1)}+a_{1}^{(2)}}\left(z_{1}\right) \ldots \phi_{a_{n}^{(1)}+a_{n}^{(2)}}\left(z_{n}\right) .
\end{aligned}
$$

The structure of the coefficients is then correctly represented by

$$
\operatorname{Tr}\left(\Gamma^{[1] c_{1}} \ldots \Gamma^{[n] c_{n}} \Gamma^{5}\right)
$$

where, for all $i$,

$\Gamma_{1 / 2}^{c}=\sum_{a_{1}+a_{2}=c} \sqrt{\left(\begin{array}{c}a_{1}+a_{2} \\ a_{1}\end{array}\right)} \gamma^{a_{1}} \otimes \gamma^{a_{2}}, \quad \Gamma_{1 / 2}^{5}=\left(\gamma^{5}\right)^{\otimes 2}$.

where the indices $c_{i}$ have range $\{0, \ldots,(n-1) / \nu\}$, that is higher powers of $z_{i}$ are involved than for $\nu=1$.

It follows that the dimensions of the combined matrices produces an effective $\chi=2^{n}$. In general, an MPS representation of the LWF with filling fraction $\nu=1 / m$ for integer $m$ can be defined in terms of the matrices $\Gamma_{1 / m}^{5}=$ $\left(\gamma^{5}\right)^{\otimes m}$,

$$
\Gamma_{1 / m}^{c}=\sum_{a_{1}+\cdots+a_{m}=c} \sqrt{\frac{\left(a_{1}+\cdots+a_{m}\right) !}{a_{1} ! \cdots a_{m} !}} \gamma^{a_{1}} \otimes \cdots \otimes \gamma^{a_{m}},
$$


yielding an effective $\chi=2^{m n / 2}$. This value of $\chi$ is far above what one could expect to be the minimum from Eq. (14) so that a much more economical construction should exist. Indeed, in the case $n=2$, we have found the following construction. The matrices $\Gamma_{1 / m}^{c}$ can be viewed as acting on the $m$-fold tensor product representation of $\mathrm{SU}(2)$. Define $\Upsilon_{1 / m}^{c}=G^{\dagger} \Gamma_{1 / m}^{c} G$, where $G$ is a projector onto the spin- $m / 2$ irreducible subspace contained in $(1 / 2)^{\otimes m}$ (a piece of Clebsch-Gordan matrix). After some algebra, we find that the explicit form of each matrix has just a single entry 1 in its antidiagonal, $\Upsilon_{1 / m}^{c}(c+1, n-c)=1$, and the rest is 0 . Further let $\Upsilon_{1 / m}^{5}$ denote an $m+1 \times m+1$ matrix whose diagonal elements are defined as $\left(Y_{1 / m}^{5}\right)_{i i}=(-)^{i-1}\left(\begin{array}{c}m \\ i-1\end{array}\right)$ and whose offdiagonal elements are zero. We have numerically checked $\Upsilon_{1 / m}^{c}$ and $Y^{5}$ yield an exact matrix-product representation of $\Psi_{\nu}$ in the case $n=2$ for $m=1 \ldots 15$, and we believe that they also do for arbitrary values of $m$. Note that for $n=2$, the von Neumann entropy of the state of one particle can be easily calculated for any value of $\nu=1 / \mathrm{m}$. One gets

$$
S_{1 / m}=\sum_{j=0}^{m}\left(\begin{array}{c}
m \\
j
\end{array}\right) \log _{2}\left(\begin{array}{c}
m \\
j
\end{array}\right) .
$$

Clearly the size of the new matrices is now $m+1$ instead of $2^{m}$, that is exponentially more economical. This representation has an interest as a starting point to get intuition on more involved cases. But, as such, it is not very useful in practice since the computation of a mean value $\left\langle V_{1} \otimes V_{2}\right\rangle$ requires an effort that scales as $O\left(\mathrm{~m}^{2}\right)$, which is the same as if using the Schmidt decomposition for $\Psi_{\nu}$.

For some filling fractions $\nu$, the foregoing analysis can be easily extended to study excited states of systems whose ground state is described by an LWF. Let us consider the simplest case of one quasiparticle localized at a position $z_{A}$. The corresponding wave function reads [6]

$$
\Psi_{\nu}^{\left[z_{A}\right]}\left(z_{1}, \ldots, z_{n}\right)=\prod_{i=1}^{n}\left(z_{i}-z_{A}\right) \Psi_{\nu}\left(z_{1}, \ldots, z_{n}\right) .
$$

Now since

$$
\prod_{i=1}^{n}\left(z_{i}-z_{A}\right)=\frac{\epsilon^{i_{A}, i_{1} \ldots i_{n}} z_{A}^{i_{A}} z_{1}^{i_{1}} \ldots z_{n}^{i_{n}}}{\epsilon^{i_{1} \ldots i_{n}} z_{1}^{i_{1}} \ldots z_{n}^{i_{n}}}
$$

we have that $\Psi_{\nu^{\prime}}^{\left[z_{A}\right]}\left(z_{1}, \ldots, z_{n}\right)=$ $\boldsymbol{\epsilon}^{i_{A}, i_{1} \ldots i_{n}} z_{A}^{i_{A}} z_{1}^{i_{1}} \ldots z_{n}^{i_{n}} \Psi_{\nu}\left(z_{1}, \ldots, z_{n}\right)$, with $\nu^{\prime}=\nu /(\nu+1)$. With calculations akin to the ones performed above, an MPS representation of $\Psi_{\nu^{\prime}}^{\left[z_{A}\right]}$ is easily derived. Defining $\Gamma^{0}\left(z_{A}\right)=\sum_{i_{A}=0}^{n} \gamma^{i_{A}} z_{A}^{i_{A}} \otimes 1$, we have (up to normalization):

$\Psi_{\nu^{\prime}}^{\left[z_{A}\right]}\left(z_{1}, \ldots, z_{n}\right)=\operatorname{Tr}\left(\Gamma^{0}\left(z_{A}\right) \Gamma^{a_{1}} \ldots \Gamma^{a_{n}} \Gamma_{5}\right) \phi_{a_{1}}\left(z_{1}\right) \ldots \phi_{a_{n}}\left(z_{n}\right)$.
MPS representations of $m$-quasiparticle excited states can be computed likewise.

In summary, we have computed the von Neumann entropy of a Laughlin gas for any bipartition $(k, n-k)$ of the system under study and for various values of the filling fraction $\nu$. We have seen that this entropy grows at most linearly with $n$ and logarithmically with $\nu$. Since this quantity can be related to the difficulty of numerically simulating the gas, this computation sheds new light on why such systems are so difficult to study. Next, we have provided an MPS representation of the Laughlin wave function, that is asymptotically optimal in the case $\nu=$ 1. We believe this representation can be exploited to compute various quantities related to the LWF, such as its norm. This will be the subject of further investigation.

We thank J.J. García-Ripoll for helpful discussions, L. Tagliacozzo for some preliminary numerical checks, and J. M. Escartin for a critical reading of this manuscript. This work has been supported by MEC (Spain), QAP (EU), and Grup consolidat (Generalitat de Catalunya).

Note added. - After completion of our work, we became aware of Ref. [11] whose results have some overlap with ours.

[1] K. von Klitzing, G. Dorda, and M. Pepper, Phys. Rev. Lett. 45, 494 (1980).

[2] D. C. Tsui, H. L. Stormer, and A. C. Gossard, Phys. Rev. Lett. 48, 1559 (1982).

[3] R. B. Laughlin, Phys. Rev. B 23, 5632 (1981); B. I. Halperin, Phys. Rev. B 25, 2185 (1982); P. Streda, J. Kucera, and A.H. MacDonald, Phys. Rev. Lett. 59, 1973 (1987); J. K. Jain and S. A. Kivelson, Phys. Rev. Lett. 60, 1542 (1988); M. Büttiker, Phys. Rev. Lett. 57 1761 (1986); M. Büttiker, Phys. Rev. B 389375 (1988).

[4] R. B. Laughlin, Phys. Rev. Lett. 50, 1395 (1983).

[5] D. Yoshioka, The Quantum Hall Effect (Springer-Verlag, Berlin, 2002).

[6] B. Paredes, P. Fedichev, J. I. Cirac, and P. Zoller, Phys. Rev. Lett. 87, 010402 (2001).

[7] G. Vidal, Phys. Rev. Lett. 93, 040502 (2004); G. Vidal, cond-mat/0605597; F. Verstraete, D. Porras, and J.I. Cirac, Phys. Rev. Lett. 93, 227205 (2004); F. Verstraete, J. J. García-Ripoll, and J. I. Cirac, Phys. Rev. Lett. 93, 207204 (2004); D. Pérez-García, F. Verstraete, M. M. Wolf, and J. I. Cirac, quant-ph/0608197.

[8] B. Zeng, H. Zhai, and Z. Xu, Phys. Rev. A 66, 042324 (2002).

[9] P. Di Francesco, M. Gaudin, C. Itzykson, and F. Lesage, Int. J. Mod. Phys. A 9, 4257 (1994).

[10] M.E. Peskin and D. V. Schroeder, An Introduction to Quantum Field Theory (Addison-Wesley, Reading, MA, 1995).

[11] Masudul Haque, Oleksandr Zozulya, and Kareljan Schoutens, preceding Comment, Phys. Rev. Lett. 98, 060401 (2007). 